\section{Complementary \\ Medicine Research \\ Practice|Methods|Perspectives}

Complement Med Res 2019;26:148-149

DOI: 10.1159/000501063
Received: May 21, 2019

Accepted: May 21, 2019

Published online: 28. Mai 2019

\title{
Prof. Dr. med. Peter F. Matthiessen - † 30. April 2019
}

Peter Matthiessen ist gegangen. Nicht "von uns", wie man es vielleicht konventionell formulieren würde. Dafür hat er zu viele Spuren bei allen hinterlassen, die sich in den letzten 40 Jahren mit der Komplementärmedizin beschäftigt haben, die zu Beginn seines Wirkens noch als "Unkonventionelle Medizinische Richtungen" bezeichnet wurden, was sich im Ansatz ähnlich noch in der Bezeichnung der "besonderen Therapierichtungen" im Bundesarzneimittelgesetz wiederfindet. "Unkonventionell" und "besonders", das sind auch die Attribute, die zur Person von Peter Matthiessen passen. Stets war er in der Scientific Community derjenige, der durch präzise Analysen und mit erkenntnistheoretischem Blick den Dingen auf den Grund ging. Aber eben nicht im Mainstream treibend, sondern in der ihm ganz eigenen Herangehensweise, rhetorisch brillant und philosophisch fundiert. Da konnte sein Vortrag auch schon einmal länger werden als vom Veranstalter geplant.

Und er glaubte an seine Sache und die Menschen, die er begeistern wollte: Sowohl bei den Patienten als auch in der Wissenschaft war sein Credo immer: nicht vor dem Ertrinken retten, sondern zum Schwimmen befähigen. Er selbst scheute dabei auch die tiefsten Gewässer und tückischen Strömungen des Wissenschaftsbetriebes nicht. In Auseinandersetzungen mit Skeptikern und politischen Entscheidungsträgern, aber auch mit seinen Kollegen und Mitarbeitern ging er Konflikten nicht aus dem Weg. Dass dies auch schmerzhaft war und zumal tiefe Furchen hinterließ, das nahm er auch für sich in Kauf. Und dass der andere "auch recht haben könnte", war eine später von ihm benutzte Redewendung, die vielleicht bewusst im Konjunktiv gehalten war. Er pflegte darauf hinzuwei-

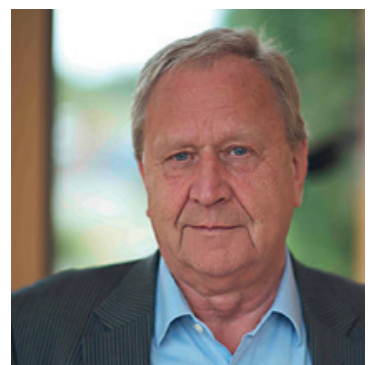

Peter F. Matthiessen

sen, dass das die Kurzfassung war, die Gadamer am Ende seines Lebens für die Essenz seiner Hermeneutik einem Journalisten mitgegeben hatte.

Die Spuren, die er hinterlassen hat, sind vielfältig. Neben den Projektbegleitungen des Förderschwerpunkts "Unkonventionelle Medizinische Richtungen" (UMR) und "Unkonventionelle Methoden der Krebsbekämpfung" (UMK) im Auftrag des Bundesforschungsministeriums (BMBF, damals BMFT), in denen von 1986 bis 1996 insgesamt 31 Projekte aus den unterschiedlichsten Bereichen mit einem Budget von mehr als 25 Mio. DM gefördert wurden und aus dem 1992 eine erste Bestandsaufnahme zur Forschungssituation entstand, wurde von 1993 bis 1998 auch die erste europäische Initiative, das COST-B4 Projekt, maßgeblich von ihm geprägt, aus dem 1999 auch die "Recommendations for Developing Unconventional Research" hervorgingen. In diese Zeit fällt auch die von ihm initiierte Publikation im Deutschen Ärzteblatt, in der ein "Brückenschlag zwischen konventioneller und alternativer Medizin" gefordert wurde und die die Idee der Integrativen Medizin bereits vorweg-

\section{KARGER}

(C) 2019 S. Karger AG, Basel
Prof. Dr. Thomas Ostermann

Department für Psychologie und Psychotherapie, Universität Witten/Herdecke Alfred-Herrhausen-Straße 50

DE-58448 Witten (Deutschland)

E-Mail thomas.ostermann@uni-wh.de 
nahm. Und auch bei der Etablierung der ersten Modellabteilungen für Naturheilverfahren in Hattingen-Blankenstein und an den Kliniken Essen-Mitte, die zu dieser Zeit entstanden, war er beteiligt. Mittlerweile in der Position des Lehrstuhls für Medizintheorie und Komplementärmedizin widmete er sich im Auftrag des Eidgenössischen Bundesamtes für Sozialversicherungen im Projekt Evaluation Komplementärmedizin (PEK) der Erstellung von Health Technology Assessments (HTAs) u.a. für die Homöopathie. Und auch das erste DFG-Projekt zur Komplementärmedizin, die Entwicklung einer offenen bibliografischen Datenbank für Komplementärmedizin, die als CAMbase noch immer existiert, wurde von ihm 2003 initiiert.

Sein Herzensanliegen aber war das Dialogforum Pluralismus (DPM), das er seit 2000 gemeinsam mit JörgDietrich Hoppe, dem damaligen Präsidenten der Bundesärztekammer, initiierte und von 2014 bis zuletzt als Sprecher leitete. Hier war der Raum für einen konstruktiven Diskurs zwischen konventionell und komplemen- tär orientierten Ärzten. Im Eckpunktepapier des DPM 2016 heißt es dazu: "Das Dialogforum Pluralismus in der Medizin hat in dieser Zeit Voraussetzungen geschaffen, um an einem neuen Selbstverständnis der Medizin aus verschiedenen Perspektiven arbeiten zu können. Für die praktische Ausübung des Arztberufes ist ein solches Selbstverständnis unverzichtbar! Dabei wurden auch die Zusammenarbeit und Integration von konventioneller Medizin und komplementären medizinischen Richtungen im Sinne einer evidenzbasierten Integrativen Medizin gefördert."

Viele andere Projekte, die hier nicht genannt wurden, hat Herr Matthiessen begleitet. Er hat die Menschen um ihn herum und den Bereich der Integrativen Medizin wie kaum ein anderer in Deutschland geprägt. Viele, da bin ich mir sicher, werden wissen, wofür "es einstmals gut gewesen ist". Jetzt ist er gegangen, und ich wünsche ihm von Herzen:

Komm gut an, lieber Chef Thomas Ostermann 